\title{
INDICADORES DE ENFERMEDADES NO COMUNICABLES EN ADOLESCENTES MEXICANOS EN RELACIÓN CON NIVEL SOCIOECONÓMICO E ÍNDICE DE MARGINACIÓN
}

\author{
NON-COMMUNICABLE DISEASES INDICATORS IN MEXICAN ADOLESCENTS RELATED TO SOCIOECONOMIC \\ LEVEL AND MARGINALIZATION INDEX
}

\author{
Vidal-Batres Marisol ${ }^{1}$, Galván Almazán Gicela de Jesús ${ }^{2}$, Vargas-Morales Juan Manuel ${ }^{3}$, Cossío-Torres Patricia \\ Elizabeth ${ }^{2}$, Aradillas-García Celia².
}

Universidad Autónoma de San Luis Potosí: 1 Coordinación para la Innovación y Aplicación de la Ciencia y Tecnología, 2 Facultad de Medicina, 3 Facultad de Ciencias Químicas. México.

\section{RESUMEN}

Introducción. El nivel socioeconómico es un determinante en la exposición de riesgos para la salud. Su relación con enfermedades no comunicables en países de ingreso mediano-bajo es poco conocida. Junto con el índice de marginación, se relacionan con la letalidad de la COVID-19. Objetivo: Conocer si el índice de marginación y nivel socioeconómico están asociados con factores de riesgo de enfermedades no comunicables. Material y Método: Estudio analítico transversal, 409 participantes de 10 a 20 años de edad de municipios con alta y muy baja marginación. Se midió peso, talla, presión arterial, glucosa y perfil lipídico. También, se aplicó el cuestionario AMAI. Resultados: Resistencia a insulina, presión arterial sistólica elevada y sobrepeso, y obesidad fueron las variables con mayor prevalencia. Se encontraron diferencias entre nivel socioeconómico e índice de marginación. A mayor nivel socioeconómico cifras mayores de glucosa, y a mayor marginación mejor perfil lipídico y cifras menores de glucosa. Conclusiones: Hay una elevada prevalencia de estos factores de riesgo resaltando la necesidad de intervenciones para su monitoreo y manejo. Poblaciones más vulnerables tienen mejores condiciones de salud comparadas con poblaciones de nivel socioeconómico alto. Estos resultados consistentes con diferentes estudios en países de ingreso mediano y bajo.

Palabras Clave: Enfermedades no comunicables, nivel socioeconómico, índice de marginación, adolescentes.

\section{ABSTRACT}

Introduction: Socioeconomic level is a determinant factor of exposure to health risks. Little is known about the relationship between non-communicable diseases in low-middle-income countries. Marginalization index and socioeconomic level are associated with COVID-19 lethality. Objective: Know if marginalization index and socioeconomic level are associated with risk factors for non-communicable diseases. Material and method: A cross-sectional study with 409 participants between 10 to 20 years' old who live in municipalities with high and very low marginalization index. Weight, height, blood pressure, fasting glucose, and lipidic profile were measured. In addition, the AMAl questionnaire was applied. Results: Insulin resistance, elevated systolic blood pressure, and overweight, and obesity were the variables more prevalent. Differences between comorbidities, socioeconomic level, and marginalization index were found. At a higher socioeconomic level, higher values of fasting glucose. At a greater marginalization index, better levels of lipidic profile and lower fasting glucose levels were found. Conclusion: There is an elevated incidence of these risk factors, highlighting the need for interventions to monitor and manage these diseases. The population in more vulnerable situations had better health conditions than those with higher socioeconomic level; these findings were consistent with middle- and low-income countries.

Key words: Non-communicable diseases, socioeconomic level, marginalization index.

Correspondencia: Celia Aradillas-García celia@uaslp.mx

Recibido: 11 de mayo 2021, aceptado: 12 de agosto 2021

(C) Autor2021

(c) (i)

DOI: https://doi.org/10.29105/respyn20.4-2

Citation: Vidal-Batres M., Galván Almazán G.J., Vargas-Morales J.M., Cossío-Torres P.E., Aradillas-García

C. (2021) Indicadores de enfermedades no comunicables en adolescentes mexicanos en relación con nivel socioeconómico e índice de marginación. Revista Salud Pública y Nutrición, 20 (4), 11-21. 


\section{Artículo Original}

\section{Introducción}

Las enfermedades no comunicables (ENC) son el resultado de una combinación de factores genéticos, psicológicos, ambientales, y de comportamiento según la World Health Organization (WHO,2018). Llevar un estilo de vida con una dieta poco saludable, sedentarismo, alto consumo de alcohol etc. está estrechamente vinculado con el sobrepeso y obesidad; existe una asociación directa con estos factores de riesgo para el desarrollo ENC (Kimokoti y Millen, 2016). Entre estas están: la enfermedad cardiovascular (ECV), hipertensión (HTA), cáncer, asma, y diabetes mellitus tipo 2 (DM2). Estas son las principales causas de muerte a nivel global $(71 \%)$ afectando principalmente a los países de ingreso mediano y bajo (PIMB) (Allen et al., 2017; WHO, 2018). Además, como es bien conocido, los hábitos como el consumo de tabaco, ingesta elevada de sodio, alcohol e inactividad física son factores de riesgo para desarrollarlas (WHO, 2018).

Según la Encuesta Nacional de Salud y Nutrición 2018 (ENSANUT), 38.4\% de los adolescentes presentan sobrepeso u obesidad, prevalencia que continúa aumentando (Shamah-Levy et al., 2020).

México se encuentra en los primeros lugares a nivel mundial de sobrepeso y obesidad infantil; a pesar de esto la ENSANUT no evalúa la prevalencia de indicadores bioquímicos y clínicos en esta población (Dávila-Torres, González-Izquierdo, y Barrera-Cruz, 2015). Se ha estimado que la prevalencia nacional de HTA en adolescentes es del $5.5 \%$ y $6.4 \%$ en hombres y mujeres respectivamente (Rosas-Peralta et al., 2015). Estudios en Latinoamérica reportan valores que van del 4.3 al $14.9 \%$ en esta población (Ruilope, Nunes Filho, Nadruz, Rodríguez Rosales y VerdejoParis, 2018). En cuanto al perfil lipídico, Bibiloni et al. (2016) reportan la prevalencia de alguna dislipidemia en el $48.8 \%$ de los adolescentes mexicanos principalmente bajos niveles de colesterol de alta densidad (C-HDL), y triglicéridos (TAG) elevados.

México es un país de mediano-alto ingreso según la clasificación del Banco Mundial, este clasifica las economías del mundo en 4 grupos: alto, medianoalto, mediano-bajo y bajo. Esta clasificación se basa en el ingreso nacional bruto per cápita (World Bank, 2018). La relación entre nivel socioeconómico (NSE) y las ENC es bien conocida en países de alto ingreso, pero no en PIMB (Allen et al., 2017). Es de importancia internacional conocer el comportamiento de las ENC en estos países, ya que la prevalencia de las ENC como ECV, HTA y DM2 ha aumentado en los PIMB de acuerdo a la Organización mundial de la salud (OMS, 2019, 2020). En México, también se ha identificado que los factores sociales como en el NSE, y el nivel de marginación se relacionan con la gravedad y mortalidad de la COVID-19 (Ortiz-Hernández y Pérez-Sastré, 2020).

La marginación se ha definido como un fenómeno multidimensional que se asocia a la carencia de oportunidades sociales, la ausencia de capacidades para adquirirlas o generarlas, y también a privaciones e inaccesibilidad a bienes y servicios fundamentales para el bienestar, según el Consejo Nacional de Población (CONAPO) (CONAPO, 2013).

El CONAPO desarrolló el índice de marginación que permite diferenciar las localidades de acuerdo con las carencias que padecen. En México $31.1 \%$ de las entidades federativas se encuentran en un grado de marginación alto o muy alto. Por ende, diversos grupos sociales son excluidos a nivel bienestar, recursos, capacidades y desarrollo. Diferenciar las entidades federativas y municipios según su grado de marginación es de gran utilidad dado que permiten identificar las áreas que se enfrentan a una elevada vulnerabilidad social y las desventajas que esta representa, por ejemplo, a nivel salud (CONAPO, 2015).

Por otro lado, El NSE es una determinante de la probabilidad de que los individuos se expongan a factores de riesgo ambientales y otros factores de riesgo para la salud (Kollia et al., 2016). Se han asociado conductas de riesgo como el consumo de tabaco, alcohol, y mala dieta; así como un acceso limitado a servicios de salud y poco control de las ENC en un bajo NSE. También se ha asociado positiva $y$ negativamente con el riesgo cardiovascular, morbilidad y mortalidad (Kollia et al. 2016).

Poco se conoce sobre la influencia que tiene el NSE en la salud cardiovascular (Riva, Larsen, y Bjerregaard, 2016). Un estudio en Grecia, país de alto ingreso detectó que un bajo NSE se asociaba con DM2 y con hipercolesterolemia, pero no con 
obesidad e HTA. Sin embargo, se asoció un bajo NSE como factor de riesgo a 10 años para ECV (Kollia et al. 2016). Otro estudio en Groenlandia, encontró que un NSE alto está asociado con mejor presión arterial (PA) tanto sistólica (PAS) como diastólica (PAD) (Riva et al., 2016).

Rarau et al. (2019) en Papúa Nueva Guinea, país de ingreso mediano bajo, encontraron factores de riesgo como un bajo consumo de frutas y verduras, alto consumo de azucares, sal, comida frita y alcohol, así como obesidad, HTA y bajos niveles de C-HDL en el grupo con más años de educación. Además, al comparar en grupos de mayor bienestar con menor bienestar, se encontró mayor consumo de azúcar, obesidad, HTA y niveles elevados de hemoglobina glucosilada (HbA1c) en el grupo con mayor bienestar. Asimismo, un metaanálisis evaluó la relación de DM2 con el NSE y encontró una relación positiva con la prevalencia de diabetes en países en desarrollo. Sin embargo, ocurrió lo contrario en los países desarrollados donde hubo una asociación negativa (Xu, Yu, Yin, Zheng, Li, 2017).

La actual pandemia por el coronavirus SARS-CoV-2 ha resaltado los riesgos de padecer algunas ENC. Como se ha descrito ampliamente, la obesidad, DM2 e HTA han contribuido a aumentar la letalidad durante el padecimiento por la COVID-19. Ya se han publicado las primeras implicaciones para nuestro país, sobre la elevada prevalencia de ENC frente a la COVID-19 (Denova-Gutiérrez et al., 2020).

El objetivo del estudio fue conocer si el índice de marginación y el nivel socioeconómico están asociados con el sobrepeso, obesidad, resistencia a la insulina (RI), presión arterial alterada, prediabetes, y dislipidemia; ya que son comorbilidades que aumentan el riesgo de desarrollar ENC (HTA, DM2, ECV), en esta población de estudio compuesta de adolescentes que pertenecen a un país de ingreso mediano alto.

\section{Material y Método}

Se realizó un estudio transversal analítico en 409 adolescentes entre los 10 y 20 años de edad que asistían a escuelas públicas de nivel básico, medio, y medio superior, en municipios de muy baja y alta marginación, en el estado de San Luis Potosí, México (figura 1).

\section{Muestra}

Fue estratificada a partir de los datos oficiales del Instituto Nacional de Estadística y Geografía (INEGI), se eligieron los municipios con más alto (San Luis Potosí y Matehuala) y bajo (Ahualulco, Villa de Guadalupe y Moctezuma) índice de marginación de la zona centro y altiplano del estado. Posteriormente, se eligieron las escuelas de acuerdo con los siguientes criterios de inclusión: escuelas públicas del turno matutino y nivel educativo de primaria, secundaria y bachillerato.

Los criterios de inclusión fueron, ser mexicano, asistir a una de las escuelas muestreadas, carta de consentimiento informado firmada por padres o tutores y el asentimiento del participante en el momento de realizar la toma de muestras. Los criterios de exclusión fueron, presentar una enfermedad diagnosticada, como DM2, HTA o alguna dislipidemia, el consumo de un medicamento que alterare los valores bioquímicos, embarazo.

Figura 1. Mapa de municipios de muy bajo y alto índice de marginación muestreados en el estado de San Luis Potosí, México

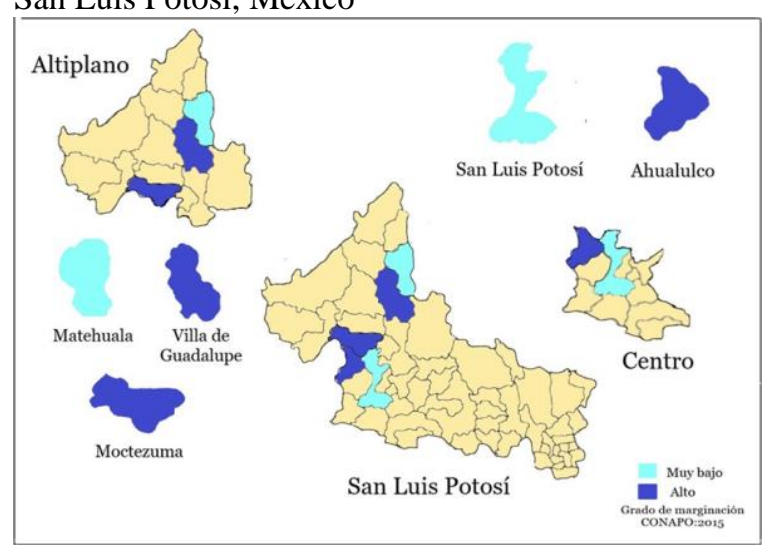




\section{Artículo Original}

\section{Variables}

Se realizó un examen clínico por aparatos y sistemas a cada participante el cual constaba de a) mediciones antropométricas (peso y talla), b) mediciones clínicas $(\mathrm{PA}), \mathrm{c})$ toma de muestra sanguínea con ayuno previo de 12 horas para determinar parámetros bioquímicos, (glucosa y perfil lipídico) y d) cuestionario AMAI 10x 6 del año 2011 para medir el NSE.

\section{a) Mediciones antropométricas}

Para evaluar el peso se utilizaron básculas electrónicas calibradas TANITA UM-081. Se pidió a los participantes que subieran a la báscula con la menor cantidad de ropa posible y sin zapatos tomándolo al $0.1 \mathrm{~kg}$ más cercano según la Norma Oficial Mexicana-047-SSA2-2015 (NOM-047SSA2, 2015). Para la talla se utilizaron estadímetros móviles marca Seca 213, $205 \mathrm{~cm}$. Se colocaron formando un ángulo de $90^{\circ}$. Se cuidó que la posición fuera firme de pie, apoyando los talones, pantorrillas, glúteos, espalda y cabeza en la pared esta se colocó de acuerdo al plano de Frankfort (Fernández, Redden, Pietrobelli y Allison, 2004). Posteriormente se calculó el índice de masa corporal (IMC) con la fórmula de Quetelet (kg/m2), mediante las puntuaciones $\mathrm{Z}$ de IMC para la edad de acuerdo con la edad y sexo, mediante el programa WHO Anthro Plus. Se utilizaron los criterios utilizados por OMS, Desnutrición ( $<-2$ puntuaciones Z), Normopeso (<-1 a 0.99 puntuaciones Z) y sobrepeso/obesidad ( $>1$ puntuaciones Z) (WHO, 2007).

\section{b) Mediciones clínicas}

La PA fue medida por profesionales de la salud capacitados de acuerdo con un protocolo común adaptado de los procedimientos recomendados por la American Heart Association (Whelton et al. 2018). Se utilizó un medidor portátil marca OMRON modelo HBP-1300. Se emplearon tres tamaños de brazaletes de acuerdo con la circunferencia braquial de cada participante, la medición fue tomada en el brazo derecho, los participantes permanecían sentados y relajados durante 5 minutos antes de la medición.

La interpretación de las medidas de PA se basó en las tablas de PA del National High Blood Pressure Education Program Working Group on High Blood Pressure; las cuales consideraban el sexo, edad y talla para su análisis, el diagnóstico de PA elevada se realizó con un percentil >90 tanto para la PAS como
PAD (Deparment of Health and Human Services, 2005).

\section{c) Parámetros bioquímicos}

Los parámetros bioquímicos incluyeron: glucosa en ayuno y perfil lipídico. Las muestras de sangre (6 $\mathrm{mL}$ ) se extrajeron con un sistema BD Vacutainer con previo ayuno de 12 horas. La glucosa en sangre fue determinada por el método de glucosa oxidasa peroxidasa GOD-PAP. Se separó el suero para las determinaciones del perfil lipídico.

Se definió prediabetes, a valores mayores a 100 $\mathrm{mg} / \mathrm{dL}$ según la American Diabetes Association (ADA, 2017). Los puntos establecidos para dislipidemia fueron colesterol total (CT) $>170$ $\mathrm{mg} / \mathrm{dL}$, colesterol de baja densidad (C-LDL) $>110 \mathrm{mg} / \mathrm{dL}, \mathrm{C}-\mathrm{HDL}<45 \mathrm{mg} / \mathrm{dl}, \mathrm{TAG}>130 \mathrm{mg} / \mathrm{dl}$ (Expert Panel on Integrated Guidelines for Cardiovascular Health and Risk Reduction in children and adolescents, 2011).

El índice de triglicéridos y glucosa en ayuno (TyG) se determinó de acuerdo con la siguiente formula $\mathrm{Ln}$ $[\mathrm{TAG}(\mathrm{mg} / \mathrm{dL})$ X Glucosa $(\mathrm{mg} / \mathrm{dL})]$ / 2, GuerreroRomero et al. (2016) evaluaron este índice en población mexicana para identificar la RI. La RI se presenta antes del desarrollo de DM2 siendo esta también un factor de riesgo para el desarrollo de ENC. Se definió RI cuando se presentaban valores $\geq 4.44$ (Locateli et al., 2019).

\section{d) Nivel socioeconómico}

En México, la Asociación Mexicana de Agencias de Inteligencia de Mercado y Opinión (AMAI) desarrollo un modelo de estimación, clasificando a los hogares en diferentes NSE. Se aplicó el cuestionario del año 2011 con la Regla 10x6 que era el más actual en ese momento.

Este considera variables como número de habitaciones, tipo de piso, baños, regaderas, focos, autos, tipo de estufa en la casa, así como la educación del principal proveedor del hogar (AMAI, 2018). Se tomó como alto NSE los grupos $\mathrm{A} / \mathrm{B}$ y $\mathrm{C}+$, mediano NSE: C, C- y D+ y bajo NSE a los grupos D, y E.

El nivel $\mathrm{A} / \mathrm{B}$ en su mayoría son viviendas propias, con sistema óptimo de sanidad, dos automóviles, por lo general el jefe de familia tiene estudios universitarios de posgrado. En el nivel $\mathrm{C}+, 2 / 3$ partes de las viviendas son propias, con un sistema óptimo 
de sanidad, en promedio cuentan con 1 o 2 automóviles y el jefe de familia tiene estudios universitarios. El nivel C, 2/3 partes tienen vivienda propia, casi todas cuentan con agua y sanidad, la educación del jefe de familia en promedio es preparatoria. El nivel D+ son casas pequeñas y la mitad propias, solo $1 / 4$ tiene automóvil, la escolaridad en promedio es secundaria, y la mayor parte de su gasto lo invierten en alimentos, transporte, y pago de servicios. El nivel D son casas pequeñas y la mitad propias, 1 de cada tres no tienen agua y 1 de cada 4 no tienen baño y la escolaridad en promedio es primaria. Finalmente, el grupo $\mathrm{E}$ son casas pequeñas donde $2 / 3$ partes tienen baño, la mayoría tiene que salir para conseguir agua, 2/3 cuentan con estufa, la escolaridad en promedio es primaria incompleta y la mayor parte de su gasto se invierte en alimentos y pago de servicios.

\section{Consideraciones éticas}

Los padres o tutores estuvieron presentes durante la recolección de todas las variables. Los datos se manejaron de manera confidencial. El estudio fue avalado por el Comité de Ética de Secretaría de Salud de San Luis Potosí.

\section{Análisis estadístico}

Los datos se analizaron mediante estadística descriptiva, las variables discretas se reportaron como porcentajes o frecuencias, y las continuas como medias y desviación estándar. La variable del NSE se dividió en tres categorías NSE alto (A/B Y C), mediano (C, C-, D+), bajo (D y E) y se tomó como variable categórica. En las variables continuas se utilizó la prueba de Kolmogórov-Smirnov (K-S) para evaluar la normalidad de los datos; de acuerdo con esto se compararon las medias de 3 grupos con la prueba de Kruskal-Wallis y de dos grupos con la prueba $U$ de Mann-Whitney. Se consideró estadísticamente significativo una $\mathrm{p}<0.05$. Se utilizó el programa IBM SPSS Statistics versión 23.

\section{Resultados}

Se evaluaron 409 participantes de los cuales el $43.5 \%$ eran hombres y el $56.5 \%$ mujeres. La edad media era de $13.67 \pm 2.68$. Con relación al indicador antropométrico la media de la puntuación $\mathrm{Z}$ del IMC se encontraba en rangos de normopeso en ambos sexos. Para el perfil lipídico la media de todas las variables se encontraba en rangos adecuados, salvo el índice TyG que se encontraba por arriba del punto de corte establecido. Al realizar el análisis de acuerdo con el sexo, los hombres presentaban valores más altos de PAS y glucosa que las mujeres, por otro lado, las mujeres tenían una media más alta en las cifras de TAG. (ver tabla No. 1).

\begin{tabular}{|c|c|c|c|c|c|c|}
\hline & \multicolumn{2}{|c|}{ Niños (n=178) } & \multicolumn{2}{|c|}{ Niñas $(n=231)$} & \multicolumn{2}{|c|}{ Total (409) } \\
\hline & $\mathrm{M}$ & DS & $M$ & DS & $\mathrm{M}$ & DS \\
\hline Edad* & 13.2 & 2.6 & 14.1 & 2.7 & 13.7 & 2.7 \\
\hline Talla* & 1.6 & 0.1 & 1.5 & 0.1 & 1.6 & 0.1 \\
\hline Peso & 51.9 & 17.6 & 50.6 & 13.1 & 51.2 & 15.2 \\
\hline $\mathrm{ZIMC}$ & 0.3 & 1.6 & 0.3 & 1.3 & 0.4 & 1.4 \\
\hline PAS* & 120.5 & 13.7 & 116.2 & 10.1 & 118.1 & 12.0 \\
\hline PAD & 66.2 & 9.3 & 66.6 & 8.8 & 66.4 & 9.0 \\
\hline $\mathrm{CT}$ & 153.8 & 27.8 & 155.6 & 29.6 & 154.8 & 28.8 \\
\hline C-HDL & 53.8 & 10.6 & 54.1 & 11.5 & 54.0 & 11.1 \\
\hline C-LDL & 80.5 & 22.5 & 80.1 & 25.2 & 80.2 & 24.0 \\
\hline TAG* & 97.3 & 53.2 & 107.5 & 52.9 & 103.1 & 53.2 \\
\hline Glucosa* & 91.4 & 7.8 & 89.4 & 14.7 & 90.3 & 12.2 \\
\hline RI & 4.5 & 0.3 & 4.5 & 0.2 & 4.5 & 0.3 \\
\hline \multicolumn{7}{|c|}{ Fuente: Encuesta } \\
\hline \multicolumn{7}{|c|}{$\begin{array}{l}\text { M: media; DS: desviación estándar; IMC: índice de masa corporal; PAS: } \\
\text { presión arterial sistólica; PAD: presión arterial diastólica; CT: colesterol } \\
\text { total; C-HDL: colesterol de alta densidad; C-LDL: colesterol de baja densidad; } \\
\text { TAG: triglicéridos; RI: resistencia a la insulina. Se utilizó la prueba U de } \\
\text { Mann-Withney para comprarlas medias. } \\
{ }^{*} p<.05\end{array}$} \\
\hline
\end{tabular}

Nuestros resultados muestran que los factores de riesgo que tienen mayor prevalencia en esta población son la RI, PAS elevada y sobrepeso u obesidad. $58.4 \%$ de la población presentaba RI, determinada de acuerdo con el índice TyG propuesto por Guerrero-Romero et al, 2016 Por otro lado, 4 de cada 10 mostraban PAS elevada; la prevalencia de sobrepeso, y obesidad fue del $33.3 \%$. En cuanto al perfil lipídico se observaron prevalencias del $29.3 \%$ de hipercolesterolemia, del $24.2 \%$ en hipertrigliceridemia y del $21.8 \%$ en hipoalfalipoproteinemia. En total el $52.3 \%$ de la población presentaba algún indicador del perfil de lípidos alterado. Finalmente, la prevalencia de prediabetes en la población total fue del $8.8 \%$.

Además, se observó una relación entre los factores de riesgo con el nivel de marginación. A mayor NSE y menor marginación mayores cifras de PA, CT, CLDL y glucosa. Sin embargo, solo se obtuvieron diferencias significativas con las variables de CHDL, C-LDL y glucosa al compararlos con el índice de marginación (ver tabla No. 2) 
Tabla 2. Asociación entre NSE y de marginación con variables antropométricas, bioquímicas y

\begin{tabular}{|c|c|c|c|c|c|c|c|c|c|c|}
\hline & \multicolumn{6}{|c|}{ Nivel socioeconómico } & \multicolumn{4}{|c|}{ Nivel de marginación } \\
\hline & \multicolumn{2}{|c|}{ Bajo } & \multicolumn{2}{|c|}{ Medio } & \multicolumn{2}{|c|}{ Alto } & \multicolumn{2}{|c|}{ Muy bajo } & \multicolumn{2}{|c|}{ Alto } \\
\hline & $\mathrm{M}$ & DS & M & DS & M & DS & M & DS & M & DS \\
\hline $\mathrm{ZIMC}$ & 0.2 & 1.3 & 0.3 & 1.5 & 0.7 & 1.3 & 0.2 & 1.5 & 0.5 & 1.3 \\
\hline PAS & 117.3 & 10.6 & 118.0 & 12.1 & 119.3 & 12.9 & 118.8 & 11.3 & 117.0 & 12.9 \\
\hline PAD & 66.1 & 11.6 & 66.3 & 8.6 & 67.0 & 7.6 & 66.9 & 8.4 & 65.5 & 9.8 \\
\hline $\mathrm{CT}$ & 151.0 & 29.7 & 155.4 & 28.1 & 156.2 & 30.8 & 156.8 & 28.9 & 151.7 & 28.4 \\
\hline C-HDL* & 52.4 & 9.7 & 54.1 & 11.2 & 55.2 & 12.0 & 53.0 & 10.9 & 55.5 & 11.4 \\
\hline C-LDL* & 78.6 & 22.0 & 80.0 & 24.0 & 82.8 & 26.2 & 83.4 & 25.7 & 75.3 & 20.1 \\
\hline TAG & 99.9 & 46.3 & 106.5 & 55.9 & 91.4 & 46.2 & 102.2 & 50.7 & 104.4 & 57.0 \\
\hline Glucosa* & 90.0 & 7.9 & 90.1 & 13.8 & 91.7 & 7.8 & 91.0 & 7.9 & 89.2 & 16.9 \\
\hline $\mathrm{RI}$ & 4.5 & 0.2 & 4.5 & 0.3 & 4.5 & 0.2 & 4.5 & 0.2 & 4.5 & 0.3 \\
\hline
\end{tabular}

IMC: índice de masa corporal; PAS: presión arterial sistólica; PAD: presión arterial diastólica; $\mathrm{CT}$ colesterol total; C-HDL: colesterol de alta densidad; C-LDL: colesterol de baja densidad; TAG:

*Nivel de marginación $p<05$

\section{Discusión}

El objetivo del estudio fue conocer si el índice de marginación y el nivel socioeconómico están asociados con el sobrepeso, obesidad, RI, presión arterial alterada, prediabetes y dislipidemia; las cuales son comorbilidades que aumentan el riesgo de desarrollar ENC, y contribuyen a las complicaciones graves de la COVID-19. Tomando en cuenta que la población de estudio son adolescentes que pertenecen a un país de ingreso mediano-alto. Se relacionaron las variables de glucosa C-HDL y CLDL con el nivel de marginación donde a mayor marginación cifras de glucosa disminuidas y mejores valores del perfil lipídico.

La elevada prevalencia de estas comorbilidades puede favorecer el desarrollo de ENC, y las elevadas prevalencias de sobrepeso, y obesidad que presentó la población de estudio (33.3\%). De acuerdo con los resultados de Kit y cols., en 2013 evaluaron la prevalencia de HTA en niños y adolescentes de 8 a 17 años, el grupo hispano obtuvo una prevalencia del $11.5 \%$, una cifra muy por debajo de la obtenida en este estudio (Kit et al., 2015). Existen pocos estudios que evalúen la prevalencia de HTA en adolescentes mexicanos, pero se estima que 5.5\% de ellos presenten pre-hipertensión e HTA. De acuerdo con el proyecto de la Norma Oficial Mexicana PROYNOM-31-SSA2-2014, Para la atención a la salud en la infancia en cada visita médica se deben medir la PA de los niños (PROY-NOM-31-SSA2, 2014). A pesar de esta normativa se observó una elevada prevalencia de esta variable, por lo tanto, una evaluación continua de la evolución de ésta es importante. La guía americana de HTA en niños sugiere monitorear este signo mínimo $1 \mathrm{vez}$ al año a partir de los 3 años y los niños y adolescentes que presenten HTA además de una intervención en el estilo de vida, deben tener un seguimiento cada 6 meses (Flynn et al., 2017). Otros estudios como el de Simental-Mendía et al. (2019), han relacionado un elevado índice TyG con pre-hipertensión e HTA en adolescentes, dos de las variables que presentaron mayor alteración en nuestra población fueron la RI por el índice TyG y la PAS elevada.

En el presente estudio, en cuanto al perfil lipídico, se observó que 5 de cada 10 adolescentes presentaba alguna dislipidemia. En 2016 un estudio en adolescentes mexicanos reportó resultados similares con una prevalencia de dislipidemias del $48.8 \%$, las más comunes fueron hipertrigliceridemia e hipoalfalipoproteinemia (Bibiloni et al., 2016). Las de mayor prevalencia en nuestro estudio fueron hipercolesterolemia e hipertrigliceridemia. Estudios en otros países como Brasil muestran prevalencias en adolescentes que van del $20 \%$ al $35 \%$ en hipercolesterolemia, del $7.8 \%$ al $18.4 \%$ en hipertrigliceridemia, del $46-50 \%$ en hipoalfalipoproteinemia y del 3.5 al $10.9 \%$ en altos niveles de C-LDL (Bloch et al., 2016; Vizentin et al., 2018). Estas cifras son similares a las nuestras con excepción de la hipertrigliceridemia donde obtuvimos cifras más elevadas. En cuanto al C-HDL obtuvimos prevalencias menores de hipoalfalipoproteinemia que en los estudios de Bloch et al,2016 y Vizentin et al,2018.

La Norma Oficial Mexicana NOM-037-SSA2-2012, Para la prevención, tratamiento y control de las dislipidemias recomienda que el monitoreo de lípidos en sangre inicie a los 20 años, sin embargo, según la ENSANUT 2018 el $47.7 \%$ de la población mayor a 20 años no cuenta con una medición del perfil lipídico (NOM-037-SSA2,2012). Además, de acuerdo con nuestros resultados es necesario iniciar con este monitoreo desde edades más tempranas. La guía americana para la salud cardiovascular en niños $\mathrm{y}$ adolescentes recomienda iniciar este monitoreo entre los 9 y 11 años cuando los niños no presentan ningún factor de riesgo (Expert Panel on Integrated Guidelines for Cardiovascular Health and Risk Reduction in children and adolescents, 2011).

En cuanto al NSE un estudio en Brasil, país de ingreso mediano alto al igual que México, reportó que, en los hombres, a mayor NSE los niveles de CHDL estaban por debajo de los valores adecuados, lo 


\section{Artículo Original}

cual resulta similar en nuestro estudio (Espírito Santo et al., 2019). Igualmente, otro estudio en Israel reportó que a menor NSE mejores niveles en el CHDL (Interator et al., 2019). La relación entre el NSE y el perfil lipídico en países desarrollados es clara, a mayor NSE un perfil lipídico más saludable, sin embargo, en los países en desarrollo se muestra un patrón diferente donde un bajo NSE se asocia a mejor perfil lipídico (Interator et al., 2019; Kollia et al., 2016; Riva et al., 2016). Nuestro estudio concuerda con este patrón en cuanto a las variables de C-HDL y C-LDL, pero no se encontraron diferencias significativas con las demás variables del perfil lipídico.

Además, un estudio realizado en 2018 con cinco PIMB encontró que los hombres de NSE alto tenían mayor riesgo de tener sobrepeso y obesidad, así como DM2 e HTA en comparación con los hombres de bajo NSE (Ogunsina, Dibaba, y Akinyemiju, 2018). Estos resultados coinciden con los nuestros donde se encontró una diferencia de medias entre los grupos de NSE con la variable de glucosa y el grupo de NSE alto tenía cifras más elevadas; además también se encontraron diferencias estadísticamente significativas en las medias del C-HDL, C-LDL, y glucosa entre los grupos de muy baja y alta marginación donde el grupo con alta marginación tenía un perfil lípido más saludable.

Estos resultados además de ser consistentes con más estudios que muestran que en los PIMB, poblaciones con NSE más alto tienen mayor riesgo de ENC, al contrario de lo que sucede con los países de ingresos altos (Kollia et al., 2016; Ogunsina et al., 2018; Riva et al., 2016). Esté revela que la relación de NSE con ENC se da desde edades tempranas puesto que nuestra población de estudio fue de un grupo de edad de $\operatorname{los} 10$ a 20 años en comparación con otras investigaciones donde la población eran adultos mayores de 18 años de edad.

Actualmente los PIMB experimentan una rápida transición nutricional que incluye, cambios en los comportamientos alimenticios, inactividad física, y composición corporal (Popkin, 2015). En los PIMB los grupos con bajo NSE son principalmente afectados por la desnutrición, por tanto, tienen menor riesgo de presentar obesidad debido al bajo acceso a alimentos. Durante el proceso de desarrollo de estos países, se empiezan a notar cambios por ejemplo al adoptar estilos de vida occidentales, las condiciones de vida mejoran, ampliando el acceso a comestibles y cambiando a una dieta occidental con alimentos de alto aporte energético y azucares. Con relación a las políticas públicas para combatir el problema de obesidad, México fue el primer país de mediano ingreso que aprobó el impuesto a bebidas azucaradas y comida chatarra, teniendo efectos importantes en la disminución del consumo de estos productos en la población de bajo nivel socioeconómico (Popkin, 2017).

La obesidad y sus comorbilidades se han asociado con mayor riesgo de enfermedades infecciosas y virales. La epidemia del COVID-19 ha causado un alto número de muertes a nivel mundial. Reportes en población mexicana han asociado mayor riesgo de desarrollar COVID-19 severo al presentar obesidad, DM2 e HTA en comparación con los que no presentaban estas comorbilidades (Denova-Gutiérrez et al., 2020). Aunque las ENC son menos comunes en adolescentes que en adultos, ambas poblaciones tienen mayores probabilidades y condiciones de desarrollar COVID-19 severo en comparación de los que no presentan estas comorbilidades (Nogueira-deAlmeida et al., 2020). Nuestros resultados muestran una elevada prevalencia de factores de riesgo para desarrollar ENC en población adolescente. Además, los índices de pobreza y de marginación también están asociados a la letalidad y gravedad de la COVID-19. Estudios en México indican que el riesgo de hospitalización y muerte aumenta en las zonas que tiene mayores índices de pobreza o mayor marginación (Gutierrez y Bertozzi, 2020; OrtizHernández y Pérez-Sastré, 2020).

Asimismo, la obesidad y sus comorbilidades conllevan otro tipo de complicaciones metabólicas que aumentan la morbimortalidad de los adolescentes que la padecen. Las complicaciones de la DM2 en adolescentes y jóvenes se desarrollan de una forma más acelerada y agresiva que cuando está se presenta en adultos de edad media. También, presentar un perfil lipídico alterado en niños y adolescentes es un indicador temprano de riesgo cardiovascular y síndrome metabólico. Además, los niños con obesidad tienen un mayor riesgo de también presentar obesidad en la vida adulta. (Zamora-Kapoor, Fyfe-Johonson, Omidpanah, Buchwald y Sinclair, 2018). 
Es necesario comprender y conocer más sobre estas comorbilidades para realizar planes de intervención y estrategias más eficaces para proteger a la población mexicana (Rosas-Peralta et al., 2015). Además, estudios exponen que, en población infantil $\mathrm{y}$ adolescente, la obesidad tiene una alta prevalencia en casos severos de COVID-19, por ejemplo, en Canadá la obesidad fue el tercer factor de riesgo más prevalente en niños y adolescentes admitidos en cuidados intensivos después de enfermedades severas asociadas a la inmunosupresión y cáncer (Nogueira-de-Almeida et al., 2020).

\section{Limitaciones del estudio.}

Una de las limitaciones de este trabajo fue que la población de estudio incluyo solo adolescentes escolarizados. Por lo que estos resultados no representan a la población adolescente que no asiste a la escuela y que probablemente ya está inserta en el mundo laboral.

\section{Conclusiones}

Los participantes de este estudio mostraron una elevada incidencia de diferentes comorbilidades para desarrollar ENC, independientemente del NSE donde se encuentren. Esto resalta la necesidad de una intervención donde se promueva el monitoreo de la presión arterial y perfil lipídico de los adolescentes, junto con una evaluación que ayude a determinar acciones específicas para cambiar aspectos del estilo de vida que puedan estar causando estas cifras tan elevadas. Se encontraron cifras menores de glucosa y perfil lipídico más saludable a un alto nivel de marginación, mostrando que un bajo nivel de marginación es un factor de riesgo en los PIMB, resultados consistentes con diferentes estudios que evalúan en NSE y nivel de marginación en diferentes PIMB.

\footnotetext{
Agradecimientos

Este trabajo fue apoyado por los FONDOS-MIXTOS CONACYT-Gobierno del Estado de San Luis Potosí, a través del proyecto: Identificación de perfiles genéticos, proteómicos y factores de riesgo asociados a enfermedades no transmisibles y sus comorbilidades e implementación de intervenciones educativas para su prevención (FMSLP- 2014-C02251723). Se agradece a la Secretaría de Educación Pública y a todas las escuelas que participaron en este trabajo.
}

Financiamiento

CONACYT con número de registro de FMSLP2014-02-251723

\section{Bibliografía}

Allen, L., Williams, H., Townsend, N., Mikkelsen, B., Roberts, N., Foster, C., y Wickramasinghe, K. (2017). Socioeconomic status and noncommunicable disease behavioral risk factors in low-income and lower-middle-income countries: A systematic review. Lancet Globl Health, 5(3), e277-89. doi: 10.1016/S2214-109X(17)30058-X.

American Diabetes Association. (2017). Classification and diagnosis of diabetes. Diabetes Care, 40 (Supplement 1),S11-24. doi: $10.2337 / \mathrm{dc} 17-\mathrm{S} 005$.

Bibiloni, M del M., Salas, R., De la Garza, Y.E., Villarreal, J.Z., Sureda, A., y Josep, T.A. (2016). Serum lipid profile, prevalence of dyslipidemia, and associated risk factors among northern Mexican adolescents: J Pediatr Gastroenterol Nutr, 63(5), 544-49. doi: 10.1097/MPG.0000000000001325.

Bloch, K.V., Klein, C.H., Szklo, M., Kuschnir, M.C.C., Abreu, G de A., Barufaldi, L.A.,...Goldberg, T.B.L. (2016). ERICA: prevalences of hypertension and obesity in Brazilian adolescents. Rev Saúde Públ, 50(suppl 1). DOI: $10.1590 / \mathrm{s} 01518-8787.2016050006685$.

Consejo Nacional de Población. Índice absoluto de marginación 2000-2010. México, D.F. Consejo Nacional de Población 2013. Recuperado de: http://www.conapo.gob.mx/es/CONAPO/Indice _Absoluto_de_Marginacion_2000_2010

Consejo Nacional de Población. Índice de marginación por entidad federativa y municipio 2015. México, D.F. Consejo Nacional de Población 2015. Recuperado de: https://www.gob.mx/conapo/documentos/indicede-marginacion-por-entidad-federativa-ymunicipio-2015

Dávila-Torres, J., González-Izquierdo, J de J., y Barrera-Cruz, A. (2015). Panorama de la obesidad en México. Rev Med Inst Mex Seguro Soc, 53 (2),240-9 
Denova-Gutiérrez, E., Lopez-Gatell, H., AlomiaZegarra, J.L., López-Ridaura, R., ZaragozaJiménez, C.A., Dyer-Leal, D.D.,... Barquera, S. (2020). The association of obesity, type 2 diabetes, and hypertension with severe coronavirus disease 2019 on admission among Mexican patients. Obesity (Silver Spring),28(10),1826-32 doi: 10.1002/oby.22946.

Deparment of Health and Human Services. (2005). Diagnosis, Evaluation, and Treatment of High Blood Pressure in Children and Adolescentes. Recuperado de: https://www.nhlbi. nih.gov/health-topics/fourth-report-ondiagnosis-evaluation-treatment-high-bloodpressure-in-children-and-adolescents.

Espírito Santo, L.R., Thaís, O.F., Silva,C.S.O., Xavier, L.A., Reis, V.C., Mota, G.A.,... Baldo, M.P. (2019). Socioeconomic status and education level are associated with dyslipidemia in adults not taking lipid-lowering medication: A population-based study. Int Health,6, ihz089. doi:10.1093/inthealth/ihz089.

Expert panel on integrated guidelines for cardiovascular health and risk reduction in children and adolescents. (2011). Expert panel on integrated guidelines for cardiovascular health and risk reduction in children and adolescents: summary report. Pediatrics, 128(supplement), s213-56. doi: 10.1542/peds.2009-2107c.

Fernández, J.R., Redden, D.T., Pietrobelli, A., y Allison, D.B. (2004). Waist circumference percentiles in nationally representative samples of African-American, European-American, and Mexican-American children and adolescents. $J$ Pediatr, 145(4), 439-44. doi: 10.1016/j.jpeds.2004.06.044.

Flynn, J.T., Kaelber, D.C., Baker-Smith, C.M., Blowey, D., Carroll, A.E., Daniels, S.E.,...Urbina, E.M.(2017). Clinical Practice Guideline for Screening and Management of High Blood Pressure in Children and Adolescents. Pediatrics, 140(3), e20171904. doi: https://doi.org/10.1542/peds.2017-1904

Guerrero-Romero, F., Villalobos-Molina, R., Jiménez-Flores, J.R., Simental-Mendia, L.E.,
Méndez-Cruz, R., Murguía-Romero, M., y Rodríguez-Morán, M. (2016). Fasting triglycerides and glucose index as a diagnostic test for insulin resistance in young adults. Arch Med Res, 47(5), 382-87. doi: 10.1016/j.arcmed.2016.08.012.

Gutierrez, J. P., y Bertozzi, S.M. (2020). Noncommunicable diseases and inequalities increase risk of death among COVID-19 patients in Mexico. PLoS One, 15(10), e0240394. doi: 10.1371/journal.pone.0240394.

Interator, H., Brener, A., Hoshen, M., Safra, I., Balicer, R., Leshno, M.,... Lebenthal, Y. (2019). Sex, ethnicity, and socioeconomic status effect on Israeli pediatric lipid testing despite equality in National Healthcare Services. Isr Med Assoc J, 21(6),369-75.

Kimokoti, R. W., y Millen B.E. (2016). Nutrition for the prevention of chronic diseases. Med Clin of North Am, 100(6),1185-98. doi: 10.1016/j.mcna.2016.06.003.

Kit, B. K., Kuklina, E., Carroll, M.D., Ostchega, Y., Freedman, D.S y Ogden, C.L. (2015). Prevalence of and trends in dyslipidemia and blood pressure among US children and adolescents, 1999-2012. JAMA Pediatr,169(3),272. doi: 10.1001/jamapediatrics.2014.3216.

Kollia, N., Panagiotakos, D.B., Georgousopoulou, E., Chrysohoou, C., Tousoulis, D., Stefanadis, C.,...Pitsavos, C. (2016). Exploring the association between low socioeconomic status and cardiovascular disease risk in healthy Greeks, in the years of financial crisis (2002-2012): The ATTICA Study. Int J Cardiol, 223,758-63. doi: 10.1016/j.ijcard.2016.08.294.

La Asociación Mexicana de agencias de Inteligencia de Mercado y Opinión. (2018). Distribución del Nivel Socioeconómico de los Hogares según Entidad Federativa 2018. Recuperado de http://nse.amai.org/data2018/

Locateli, J.C., Lopes, W.A., Simões, C.F., de Oliveira, G.H., Oltramari, K., Bim, R.H.,... Nardo, N.J. (2019). Triglyceride/glucose index is a reliable alternative marker for insulin resistance 
in South American overweight and obese children and adolescents. J Pediatr Endocrinol Metab, 32(10),1163-70. doi: 10.1515/jpem2019-0037.

Nogueira-de-Almeida, C.A., Del Ciampo, L.A., Ferraz, I.S., Del Ciampo, L.R.L., Contini, A.A., y Ued, F da V. (2020). COVID-19 and obesity in childhood and adolescence: A clinical review. $J$ Pediatr (Rio J), 96(5),546-58. doi: 10.1016/j.jped.2020.07.001.

Ogunsina, K., Dibaba, D.T., y Akinyemiju. T.( 2018). Association between life-course socioeconomic status and prevalence of cardiometabolic risk ractors in five middle-income countries. $J$ Glob Health, 8(2),020405. doi:10.7189/jogh.08.020405.

Organización Mundial de la Salud. (2019). Hipertensión. Recuperado de https://www.who.int/es/news-room/factsheets/detail/hypertension

Organización Mundial de la Salud. (2020). Diabetes. Recuperado de https://www.who.int/es/newsroom/fact-sheets/detail/diabetes.

Ortiz-Hernández, L., y Pérez-Sastré, M.A. (2020). Inequidades sociales en la progresión de la COVID-19 en población mexicana. Rev Panam Salud Pública, 44:1. doi: 10.26633/RPSP.2020.106.

Popkin, B.M. (2015). Nutrition transition and the global diabetes epidemic. Curr Diab Rep, 15(9), 64. doi: 10.1007/s11892-015-0631-4.

Popkin, B.M. (2017). Relationship between shifts in food system dynamics and acceleration of the global nutrition transition. Nutr Rev, 75(2),73-82. doi: 10.1093/nutrit/nuw064.

Rarau, P., Pulford, J., Gouda, H., Phuanukoonon, S., Bullen, C., Scragg, R., Oldenburg, B. (2019). Socio-economic status and behavioral and cardiovascular risk factors in Papua New Guinea: A cross-sectional survey. PLoS One, 14(1), e0211068. doi: 10.1371/journal.pone.0211068.
Riva, M., Larsen, C.V.L., y Bjerregaard P. (2016). Association between individual-level and community-level socio-economic status and blood pressure among Inuit in Greenland. Int $J$ Circumpolar Health,75(1), 32757. doi: 10.3402/ijch.v75.32757.

Rosas-Peralta, M., Medina-Concebida, L.E., Borrayo-Sánchez, G., Madrid-Miller, A., Ramírez-Arias, E., y Pérez-Rodríguez, G. (2016). Hipertensión arterial sistémica en el niño y adolescente. Rev Med Inst Seguro Soc, 54 (S1), 52-66.

Ruilope, L. M., Nunes Filho, A.C.B., Nadruz, W. Jr., Rodríguez Rosales, F.F., y Verdejo-Paris, J. (2018). Obesity and hypertension in Latin America: current perspectives. Hipertens Riesgo Vasc, 35(2),70-76. doi: 10.1016/j.hipert.2017.12.004.

Secretaría de Salud de México. Norma Oficial Mexicana NOM-047-SSA2-2015, "Para la atención a la salud del Grupo Etario de 10-19 años de edad". México, DF; Diario Oficial de la Federación: agosto 12,2015

Secretaría de Salud de México. Norma Oficial Mexicana NOM-37-SSA2-2012 "Para la prevención, tratamiento y control de las dislipidemias", México, DF; Diario Oficial de la Federación: julio 13,2012

Secretaría de Salud de México. Proyecto de Norma Oficial Mexicana PROY-NOM-31-SSA2-2014, "Para la atención a la salud en la infancia". México, DF; Diario Oficial de la Federación: noviembre 25,2015

Shamah-Levy, T., Vielma-Orozco, E., HerediaHernandez, O., Romero-Martinez, M., MojicaCuevas, J., Cuevas-Nasu, L.,... RiveraDommarco, J. (2020). Encuesta Nacional de Salud y Nutrición 2018-19: Resultados Nacionales. Cuernavaca, México: Instituto Nacional de Salud Pública.

Simental-Mendía, L.E., Hernández-Ronquillo, G., Gamboa-Gómez, C.I., Gómez-Díaz, R., Rodríguez-Morán, M., y Guerrero-Romero, F. (2019). The triglycerides and glucose index is 
associated with elevated blood pressure in apparently healthy children and adolescents». Eur J Pediatr, 178(7),1069-74. doi: 10.1007/s00431-019-03392-x.

Vizentin, N.P., Santos Cardoso, P.M., Gomes Maia, C.A., Perez Alves, I., Lunardi Aranha, G. y Tavares Giannini, D. (2018). Dyslipidemia in adolescents seen in a University hospital in the city of Rio de Janeiro/Brazil: Prevalence and association. Arq Bras Cardiol, doi: 10.5935/abc. 20180254 .

Whelton, P.K., Carey, R.M., Aronow, W.S., Casey, D.E Jr., Collins, K.J., Dennison Himmelfarb, C., ...Wright, J.T Jr. (2017). ACC/AHA/AAPA/ABC/ ACPM/AGS/APhA/ASH/ASPC/NMA/PCNA guideline for the prevention, detection, evaluation, and management of high blood pressure in adults: executive summary: a report of the American College of Cardiology/American Heart Association Task Force on Clinical Practice Guidelines. Hypertension, 71,12691324. doi: 10.1161/HYP.0000000000000066.

World Bank. (2018). Clasificación de los países según el nivel de ingreso. Recuperado de http://databank.worldbank.org/data/download/sit e-content/CLASS.xlsx

World Health Organization. (2007). Growth reference data for 5-19 years. Recuperado de https://www.who.int/tools/growth-referencedata-for-5to19-years/application-tools

World Health Organization. (2018). Noncommunicable diseases. Recuperado de https://www.who.int/news-room/factsheets/detail/noncommunicable-diseases

Xu, Z., Yu, D., Yin, X., Zheng, F., y Li, H. (2017). Socioeconomic status is associated with global diabetes prevalence. Oncotarget, 8(27),4443439. doi: 10.18632/oncotarget.17902.

Zamora-Kapoor, A., Fyfe-Johonson A., Omidpanah, A., y Buchwald D. (2018). Risk factors for prediabetes and diabetes in adolescence and their variability by race and ethnicity. Preventive
Medicine, $115,47-52$. 10.1016/j.ypmed.2018.08.015 doi: 